\title{
Striatal activity in concept learning
}

\author{
CAROL A. SEGER and CORINNA M. CINCOTTA \\ Colorado State University, Ft. Collins, Colorado
}

\begin{abstract}
Striatal learning systems have been implicated in learning relationships between visual stimuli and outcomes. In the present study, the activity of the striatum during visual concept learning in humans was examined by using functional magnetic resonance imaging (fMRI). Participants performed three conceptlearning tasks and a baseline task. The participants were trained to criterion before fMRI scanning on two tasks, verbal and implicit. In the verbal task, classification could be performed on the basis of a simple verbal rule, but in the implicit task, there was no simple verbal rule. The novel-implicit learning task, in which an implicit structure was used, was not encountered by the participants before scanning. Across all three concept-learning tasks, there was significant activation in the striatum, in comparison with the baseline task. The striatum was recruited similarly in classification when the participants had different levels of expertise (novel-implicit vs. verbal and implicit) and were able to verbalize their learning to different degrees (verbal vs. implicit and novel-implicit). There was left lateral occipital activation when learning was implicit (implicit and novel-implicit), but not when learning was easily verbalized (verbal).
\end{abstract}

The basal ganglia make contributions to behaviorbeyond modulation of movement. The striatum has been found to be important for learning, when the organism must learn sensory discriminations between items associated with different responses or outcomes. Research in rats has shown that striatal lesions impair performance in tasks that require that the rat should learn to repeatedly choose the same location in a radial arm maze (Packard, Hirsch, \& White, 1989 ) or to repeatedly swim to a visible platform in the Morris water maze (Packard \& McGaugh, 1992). Research with monkeys has focused on concurrent discrimination tasks in which the monkey is trained simultaneously on 820 pairs of objects to repeatedly choose the same object from each pair. Both the tail of the caudate (FernandezRuiz, Wang, Aigner, \& Mishkin, 2001; Teng, Stefanacci, Squire, \& Zola, 2000; Wang, Aigner, \& Mishkin, 1990) and temporal lobe visual processing area TE, which provides input to the tail of the caudate (Buffalo et al., 1999; Buffalo, Stefanacci, Squire \& Zola, 1998), are required for normal performance on concurrent discrimination tasks. Conversely, visual discrimination learning is preserved when all connections from visual areas of the inferior temporal lobe other than the connection to the striatum are severed (Gaffan \& Eacott, 1995). Striatal-based learning has sometimes been termed habit learning, in contrast with memory learning, subserved by hippocampal-diencephalic neural systems (Mishkin, Malamut, \& Bachevalier, 1984). The striatal and hippocampal-diencephalic systems operate in parallel and, at times, in opposition to each other (Packard et al., 1989; Poldrack, Prabhakaran, Seger, \& Gabrieli, 1999).

Correspondence concerning this article should be addressed to C. A. Seger, Department of Psychology, Colorado State University, Ft. Collins, CO 80523 (e-mail: seger@lamar.colostate.edu).
A puzzling feature of the habit-memory distinction has been that human amnesia patients in whom the hippocampus or the diencephalon is damaged but the striatum is intact are impaired on concurrent discrimination tasks (Aggleton, Shaw, \& Gaffan, 1992; Oscar-Berman \& Zola-Morgan, 1980; Squire, Zola-Morgan, \& Chen, 1988) that monkeys with damage limited to the hippocampus can learn (Teng et al., 2000). Humans may perform these tasks in ways qualitatively different from those of other animals: $\mathrm{Hu}-$ mans tend to learn the correct object choice for each pair presented in concurrent discrimination in a single trial, whereas monkeys learn the discriminations slowly over many trials (Squire et al., 1988). Functional imaging has shown activation of the head of the caudate in a delayed matching-to-sample task with a short delay, in which the participant must choose a matching stimulus after a delay of up to $15 \mathrm{sec}$ (Elliott \& Dolan, 1999), but it is unclear whether the caudate is necessary for learning across longer delays, as is required in the concurrent discrimination task.

By making the relationships between stimuli and outcomes more complex, researchers have developed tasks that require the striatum for learning in humans. One such group of tasks consists of the probabilistic classification tasks, often instantiated as weather prediction tasks, in which participants learn to predict an outcome (rain or sunshine) on the basis of a set of cue cards. Stimulus-outcome relations in this task are probabilistic, in that each set of cards has a nonzero probability of being associated with each outcome. Amnesic patients show preserved learning during initial blocks of this task (Knowlton, Squire, \& Gluck, 1994), whereas patients with damage to the striatum owing to Parkinson's disease (Knowlton, Mangels, \& Squire, 1996) or Huntington's disease (Knowlton, Squire, et al., 1996) are impaired on this task at all stages of learning. A functional magnetic resonance imaging (f MRI) study 
in which the weather prediction task was used showed activity in the striatum during learning (Poldrack et al., 1999). Another group of tasks consists of sequence-learning tasks in which participants make speeded responses to the position of a stimulus on a computer screen; the screen locations appear in a deterministic or a probabilistic sequence. Striatal areas are active during sequence-learning tasks under implicit conditions in which participants do not become aware of the presence of the sequence (Grafton, Hazeltine, \& Ivry. 1995; Hazeltine, Grafton, \& Ivry, 1997; Honda et al., 1998); one f MRI study in which probabilistic sequences were used (Berns, Cohen, \& Mintun, 1997) specifically found that activation in the right ventral striatum was associated with a change in the probabilistic sequential contingencies that the participants were unaware of. Sequence learning is impaired in patients with damage to the basal ganglia owing to Parkinson's and Huntington's diseases (Jackson, Jackson, Harrison, Henderson, \& Kennard, 1995; Knopman \& Nissen, 1991; Willingham \& Koroshetz, 1993).

In addition to tasks involving learning complex contingencies, the striatum in humans is also involved in other visual discrimination and categorization-learning tasks. Patients with Huntington's disease are impaired on visual tasks, such as face recognition (Jacobs, Shuren, \& Heilman, 1995). Rao et al. (1997) found activation in a PET study of the right basal ganglia when participants learned via feedback to choose between two multifeatured objects on the basis of a single feature (e.g., always choose the blue item); Channon, Jones, and Stephenson (1993) found that Parkinson's disease patients were impaired on the same task. Studies in which the Wisconsin card sorting test (WCST), traditionally considered a task sensitive to frontal lobe impairment, was used have found impairment in persons with Parkinson's disease (Caltagirone, Carlesimo, Nocentini, \& Vicari, 1994; Taylor, Saint-Cyr, \& Lang, 1986), as well as in those with Huntington's disease (Weinberger, Berman, Iadorola, Driesen, \& Zec, 1988). Early PET and $\mathrm{fMRI}$ studies were not consistent in finding striatal activity during the WCST: Goldberg et al. (1998) reported left caudate and putamen activity during WCST, whereas Ragland et al. (1998), Nagahama et al. (1996), and Berman et al. (1995) did not report striatal activation. A more recent study using event-related f MRI to isolate brain activations associated with particular phases in the WCST found that the caudate was active when negative feedback was received upon rule shift and the putamen was active when the first classification decision was made in which a new rule was used after a rule shift (Monchi, Petrides, Petre, Worsley, \& Dagher, 2001).

Anatomical features of the basal ganglia indicate that the striatum may perform its role in the learning of associations between stimuli and outcomes through integration of inputs from different cortical areas and may affect responses via context-dependent inhibition of basal ganglia output. The striatum (caudate and putamen) receives excitatory input from virtually all areas of the cerebral cortex (Mink, 1996). This input is topographically organized, with some divergence (one cortical location connecting with several striatal locations) and convergence (related cortical areas all connecting with the same striatal location; Mink, 1996; Wise, Murray, \& Gerfew, 1996). In general, the head of the caudate and the anterior putamen receive input from the prefrontal cortex, and the tail of the caudate and the posterior putamen receive input from temporal and parietal areas, but frontal afferents are also sent to converge with parietal afferents in the tail (Wise et al., 1996). The output of the striatum is focused inhibition to the output structures of the basal ganglia: the internal segment of the globus pallidus and the substantia nigra pars reticulata $(\mathrm{GPi} / \mathrm{SNr})$. This specific, focused inhibition from the striatum counters diffuse excitatory input from the motor areas of the frontal lobe via the subthalamic nucleus. Output goes from the GPi/SNr to the thalamus and then back to cortex, forming loops. Several functionally and anatomically independent loops have been identified. The two loops of particular interest for concept learning are the cognitive or spatial loop, which passes through the prefrontal cortex and the head of the caudate, and the $v i$ sual loop, which passes through the inferior temporal areas and the tail of the caudate (Alexander, DeLong, \& Strick, 1986; Lawrence, Sahakian, \& Robbins, 1998; Middleton \& Strick, 1996). The cognitive loop may be involved in rule formation and rule-based judgments about stimuli (Rao et al., 1997). The visual loop is involved in visual pattern discrimination in nonhuman animals (Buffalo et al., 1999; Buffalo et al., 1998; Fernandez-Ruiz et al., 2001; Teng et al., 2000) and may perform a similar role in human visual concept learning.

The physiology of individual caudate neurons is also consistent with a possible role of the caudate in stimulusoutcome learning. Many neurons in the striatum fire in a context-dependent manner. For example, there are neurons that fire when a stimulus that is rewarded is seen and stop firing when the reward is stopped. Kimura found that more than $80 \%$ of the putamen neurons that respond phasically following a stimulus modulated their responses when the stimulus was presented in different behavioral contexts (Kimura 1992, cited in Wise et al., 1996).

Ashby, Alfonso-Reese, Turken, and Waldron (1998) proposed a neuropsychologicaltheory of concept learning, with separate roles for the striatum in explicit (verbalizable) and implicit (minimally verbalizable) concept learning. They postulated that implicit categorizationis dependent on the visual loop passing between the tail of the caudate and inferotemporal visual form processing areas. The explicit or verbal concept-learning system is dependent on the cognitive loop running between prefrontal areas and the head of the caudate, in conjunction with the anterior cingulate. Ashby et al. (1998) further postulated that the implicit and the verbal systems will both be active early in learning and will compete, with the verbal system having an initial advantage. As formulated in their COVIS model, ultimately, the more successful system will dominate the other. 
The present experiment was designed to further explore the conditions under which the striatum is recruited in concept-learning tasks. First, the effect of verbal rule use on striatal recruitment was examined by comparing activity during classification for a task in which the decision bound between concepts is easily verbalizable (verbal) with activity during classification in a task in which the bound between concepts has no simple verbal equivalent (implicit). Ashby et al.'s (1998) theory predicts that the implicit task will activate the tail of the caudate and inferotemporal areas, whereas the verbal task will activate the anterior cingulate, prefrontal areas, and the head of the caudate. The terms verbal and implicit are used here solely to indicate the relative degree to which regularities in each task can be verbalized and are not intended to imply a different contribution of hippocampal-diencephalic memory systems to the verbal and the implicit tasks (Seger, 1994a, 1994b). A PET study by Smith, Patalano, and Jonides (1998) found that brain activity can differ between verbal rulebased classification and other classification strategies. They compared rule-based classification, in which participants classified novel animal figures according to a verbal rule, with memory-based classification, in which participants memorized the concept membership of each figure. They found relatively more right frontal and bilateral parietal activity in the rule-based condition and relatively more posterior visual area activity in the memorybased condition.

The second factor examined was whether the striatum would be recruited to a different degree during early stages of learning and during skilled classification. In order to ensure that all the participants met the criteria for skilled performance on the verbal and implicit tasks, the participants were pretrained in these tasks to a criterion level performance. Early stage classification was examined by having the participants perform a new implicit conceptlearning task (novel-implicit). Previous research has found that brain activity changes across classification learning. Seger, Prabhakaran, Poldrack, and Gabrieli (2000) found right frontal and parietal activity in the early stages of learning, bilateral frontal and parietal activity in intermediate stages, and right frontal and bilateral parietal activity during skilled performance.

\section{METHOD}

\section{Participants}

The participants were 12 Stanford students, 6 male and 6 female, with an average age of 22 years (range, 19-28). The participants were right-handed and fluent speakers of English, met the criteria for MRI scanning (no metallic implants, no claustrophobia, head size compatible with the custom head coil), and were neurologically healthy (no known neurologic or psychiatric injury or disease, not taking any psychoactive medication or drugs).

\section{Materials}

Three concept-learning tasks (verbal, implicit, and novel-implicit) were developed in which participants learned to classify exemplars into two separate categories, termed $A$ and $B$ in the experiments. The stimuli were formed according to the methods described in Ashby and Gott (1988) and Ashby and Maddox (1992), in which two features of a simple geometric form were manipulated. Feature magnitudes were defined in terms of a mean value and a standard deviation and varied according to a Gaussian distribution within each category. Mean feature values were chosen so that the distributions of concept exemplars from both categories overlapped in feature space (see Figure 2 for a graphical illustration of stimuli in feature space and of the overlap between categories). Ashby and colleagues argued that the participants in their experiments learned a decision bound between the categories, so that stimuli falling on one side of the bound were classified in one category, and stimuli falling on the other side of the bound were classified in the other category. The decision bounds were mathematical functions of the magnitude of the manipulated features within each concept and could take the form of linear or quadratic functions, among others.

For the verbal, implicit, and novel-implicit tasks, the mean or prototypical stimuli for Categories A and B are shown in Figure 1, the mean values and standard deviations of the manipulated features are given in Table 1, and the decision bound and variability within and between the categories are illustrated graphically in Figure 2. In the verbal task, commensurable features (two line lengths) were manipulated, whereas in the implicit tasks (implicit and novel-implicit), incommensurable features (one line length and one angle) were manipulated; the use of incommensurable features has been shown by Ashby and colleagues to greatly reduce verbalizability (Ashby et al., 1998).

In the verbal task, the stimuli were blue rectangles in which the lengths of the sides were manipulated. The ideal decision bound between Categories A and B was a function that can be expressed as a simple verbal rule: The rectangle is an $\mathrm{A}$ if it is taller than it is wide, a B if it is wider than it is tall. For the implicit condition, the stimuli were formed of two green lines, one horizontal and one descending from the horizontal line and forming an angle with it. The manipulated variables were the length of the horizontal line and the angle between the two lines. The ideal decision bound between Category A and Category B was a function with no simple verbalizable equivalent. In the novel-implicit task, the stimuli were formed as a black circle with a line through it. The manipulated variables were the diameter of the circle and the angle of the diameter line from horizontal. As in the implicit task, the ideal decision bound between A and B had no simple verbalizable equivalent. To minimize any carryover of learning between the implicit and the novel-implicit tasks, the form of the decision bounds to be learned were different in each task. The implicit task used a linear decision bound, whereas the novelimplicit task used a quadratic decision bound.

In all tasks, there was overlap between concepts, so that ideal accuracy in discriminating between concept exemplars was $80 \%$. That is, the means of the feature values were chosen so that $20 \%$ of the stimuli generated as exemplars of each category had feature magnitudes (length or angle) that were closer to those of the other category's prototype than to those of their own category prototype. Figure 2 illustrates the overlap between category exemplars graphically. Figure 2 also illustrates the approximate ideal decision bounds between categories, which takes the form of a linear function for the verbal and implicit tasks and a quadratic function for the novel-implicit task.

For all three concept-learning tasks, 100 different stimuli were formed. Each stimulus appeared once in each block of pretraining trials and was repeated across blocks. A subset of 48 of the stimuli was presented in the two f MRI scans. On each trial during pretraining and scanning, a single stimulus was presented, and the participants pressed one key to indicate Category A and a different key to indicate Category B. During pretraining, the stimulus remained on the computer screen until the participant made a classification response; the stimulus then disappeared, and the letter A or B ap- 
A prototype

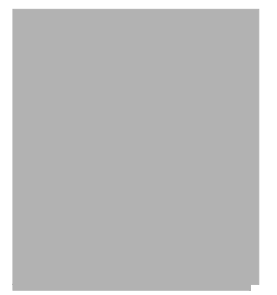

Verbal

Implicit

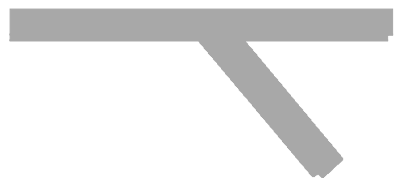

Novel-Implicit

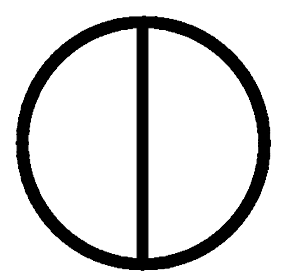

B prototype
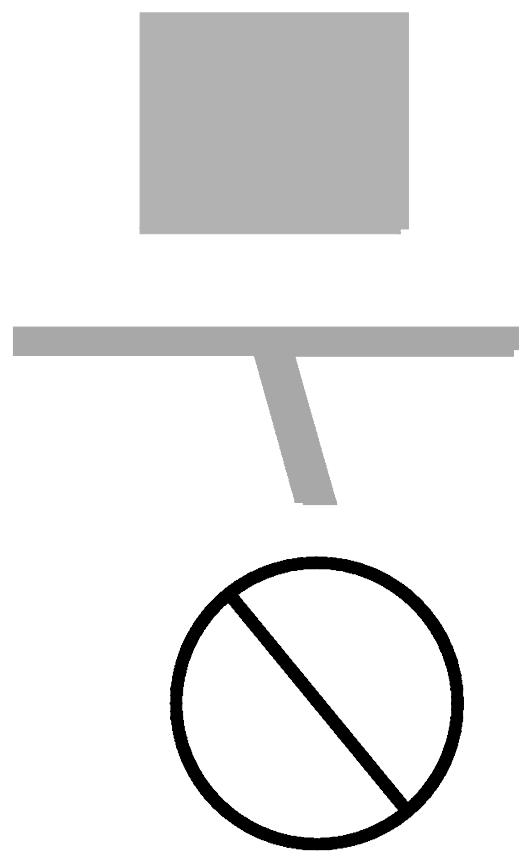

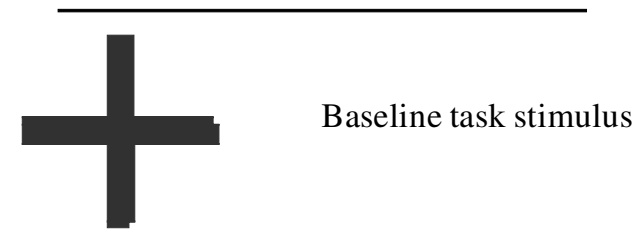

Figure 1. Prototypical stimuli for Categories $A$ and $B$ in the verbal, implicit, and novelimplicit concept-learning tasks and the baseline task stimulus. The verbal task stimuli were presented in blue, the implicit task stimuli in green, and the novel-implicit and baseline stimuli in black.

peared, to indicate the category membership of the preceding stimulus. During f MRI scanning, trials were of a fixed length of $3 \mathrm{sec}$. The stimulus remained on the screen for $2 \mathrm{sec}$, during which the participants made a classification response. The stimulus then disappeared; there was a blank screen for $250 \mathrm{msec}$, then the letter A or B for $500 \mathrm{msec}$, and then a blank screen again for $250 \mathrm{msec}$.

In addition to the three concept-learning tasks, a simple baseline task was devised to control for viewing a visual stimulus and pressing a response key. On each trial of the baseline task, the participants viewed the same stimulus (a black + , as shown in Figure 1) and pressed either response key. Baseline trials were $3 \mathrm{sec}$ in length: the stimulus for $2 \mathrm{sec}$, a blank screen for $250 \mathrm{msec}$, the letter $\mathrm{N}$ for 500 $\mathrm{msec}$, and a blank screen again for $250 \mathrm{msec}$.

\section{Procedure}

Pretraining. The participants were pretrained on the verbal and implicit tasks during a single afternoon session of $1-2 \mathrm{~h}$ preceding the evening MRI scanning session. The pretraining session was held in a behavioral testing laboratory room; the stimuli were presented with a Macintosh computer running PsyScope software (Cohen, MacWhinney, Flatt, \& Provost, 1993), and responses were collected via the computer keyboard. The participants completed blocks of 100 trials until they reached a criterion of two blocks at or above $75 \%$ accuracy. The criterion blocks did not need to be consecutive. The participants performed the verbal learning task before the implicit learning task. The interval between pretraining and MRI scanning varied from 2 to $6 \mathrm{~h}$.

fMRI image acquisition. Imaging was performed with a custombuilt whole-head coil in a $1.5 \mathrm{~T}$ whole-body MRI scanner (GE Medical Systems). Head movement was minimized by using a bite-bar formed with the participant's dental impression. Before the functional scans, two anatomical scans were performed: a coronal T1weighted localizer scan and an inplane-axial spin echo T1-weighted scan. The inplane scan consisted of nineteen 6-mm thick contiguous axial slices angled parallel to the anterior-commissure/ posteriorcommissure line. This slice prescription resulted in the entire brain's being scanned, with the exception of the bottom of the cerebellum, which fell outside the scanned region. The inplane scan had a sampling interval of $2.0 \mathrm{sec}, 1$ interleaf, TR $=2,000 \mathrm{msec}$, TE $=40$, flip angle $=87$, and field of view $(\mathrm{FOV})=220.0 \mathrm{~mm}$.

After the structural imaging, the participants participated in two identical functional scans. A T2* sensitive gradient echo spiral sequence was used for functional imaging (the spiral technique provides excellent motion immunity; Glover \& Pauly, 1992). Functional image slices were taken, using the same number and orientation of slices and the same imaging parameters as the inplane T1 anatomical scan. During each functional scan, the participants performed six blocks of each task (baseline, verbal, implicit, and novel-implicit); 
Table 1

Mean Values and Standard Deviations of the Features Manipulated in the Concept-Learning Tasks

\begin{tabular}{|c|c|c|c|c|}
\hline & \multicolumn{4}{|c|}{ Verbal Task } \\
\hline & \multicolumn{2}{|c|}{$\begin{array}{l}\text { Horizontal Side } \\
\text { Length }\end{array}$} & \multicolumn{2}{|c|}{$\begin{array}{l}\text { Vertical Side } \\
\text { Length }\end{array}$} \\
\hline & $M$ & $S D$ & $M$ & $S D$ \\
\hline Concept A & 100 & 25 & 125 & 25 \\
\hline \multirow[t]{4}{*}{ Concept B } & 125 & 25 & 100 & 25 \\
\hline & \multicolumn{4}{|c|}{ Implicit Task } \\
\hline & \multicolumn{2}{|c|}{$\begin{array}{l}\text { Horizontal Line } \\
\text { Length }\end{array}$} & \multicolumn{2}{|c|}{$\begin{array}{c}\text { Angle of Descender } \\
\text { Line } \\
\end{array}$} \\
\hline & $M$ & $S D$ & $M$ & $S D$ \\
\hline Concept A & 195 & 35 & 35 & 22 \\
\hline \multirow[t]{4}{*}{ Concept B } & 240 & 35 & 10 & 22 \\
\hline & \multicolumn{4}{|c|}{ Novel-Implicit Task } \\
\hline & \multicolumn{2}{|c|}{ Diameter Length } & \multicolumn{2}{|c|}{ Angle of Orientation } \\
\hline & $M$ & $S D$ & $M$ & $S D$ \\
\hline Concept A & 155 & 47 & 90 & 47 \\
\hline Concept B & 165 & 35 & 125 & 35 \\
\hline
\end{tabular}

Note-Side and line lengths are measured in computer screen pixels; angles are in degrees. In the implicit task, zero degrees indicates a descender pointed straight downward (i.e., $90^{\circ}$ from the horizontal line), positive numbers indicate degrees of counterclockwise rotation, and negative numbers indicate degree of clockwise rotation. In the novelimplicit task, angle is the measure of degrees counterclockwise from horizontal of the diameter line.

the baseline blocks were six trials long $(18 \mathrm{sec})$, and the experimental task blocks were eight trials long $(24 \mathrm{sec})$. Baseline blocks alternated with sets of three experimental task blocks; across the scan, the experimental tasks appeared once in each possible order. The block order was B VNI B NIV B IVN B INV B VIN B NVI, where B = baseline, $\mathrm{V}=$ verbal, $\mathrm{N}=$ novel-implicit, and $\mathrm{I}=$ implicit. Each scan lasted 9 min.

Visual stimuli were presented with a magnet-compatible projector (Resonance Technology, Van Nuys, CA) that back-projects visual images onto a screen mounted above the participant's head. A Macintosh computer, in conjunction with PsyScope software, was used to generate visual stimuli and to control experimental parameters. Responses were obtained with a fiber-optic finger switch response system that interfaces with PsyScope.

Image analysis. Image reconstruction was performed by using a gridding algorithm that resampled the raw data into a Cartesian matrix prior to processing with $2 \mathrm{~d}$ fast Fourier transform. Images were converted into volumes and preprocessed before statistical analyses were performed. Volumes were first realigned to correct for head motion, using the algorithm in SPM99. To facilitate the reporting of data in a standardized coordinate system, the volumes were normalized into the standardized anatomical space used in SPM99, which is based on that defined by the Montreal Neurological Institute (Friston, Ashburner, et al., 1995). This is similar to the standardized space defined in the Talairach and Tournoux (1988) atlas. Normalization was first performed on the anatomical inplane MRI image, using a 12-parameter affine transformation followed by an elastic deformation, and the resulting set of transformations was applied to the functional image volumes. The voxel size postnormalization was $2 \times 2 \times$ $2 \mathrm{~mm}$. The normalized and realigned functional volumes were then spatially smoothed with a Gaussian kernel at FWHM of $8.0 \mathrm{~mm}$.

With the general linear model approach (Friston, Worsley, \& Frackowiak, 1995) of SPM99, a reference waveform corresponding to the alternating conditions was constructed and convolved with an estimate of the hemodynamic response function. Corrections for multiple voxel comparisons were made by using the cluster-size method of Friston, Worsley, Frackowiak, Mazziotta, and Evans (1994). Statistical analysis of data across participants proceeded by collapsing each participant's single-session data into a set of representative contrast images, with low-frequency signal drifts and other confounds removed. The resulting images were subjected to a secondlevel group analysis, which met the requirements for random effects analysis proposed by Holmes and Friston (1998).

\section{RESULTS}

\section{Behavioral Results}

Pretraining. The participants reached criterion on the verbal task $(M=4.4, S D=1.66$, range of 3-9) in significantly fewer blocks (of 100 trials) than on the implicit task $[M=6.3, S D=1.96$, range of 3-9; $t(11)=2.64, p<$ .05]. Seven participants reached criterion on the verbal task in fewer blocks than on the implicit task, 2 reached criterion on the implicit task in fewer blocks than on the verbal task, and 3 required the same number of blocks to reach criterion on both tasks.

Scanning. On the verbal task, the participants correctly classified an average of $75.7 \%$ of the stimuli $(S D=2.4 \%$, range of $72 \%-81 \%$ ). On the implicit task, the participants correctly classified an average of $72.0 \%$ of the stimuli $(S D=3.6 \%$, range of $68 \%-81 \%)$. Thus, performance on both tasks was still near the criterion level reached during training and close to the ideal performance of $80 \%$. There was a trend toward the verbal task performance's being better than performance on the implicit task $[t(11)=2.2$, $p=.054]$.

There was evidence that the participants were classifying in accordance with the ideal decision bound in both tasks. On the verbal task, the participants classified $89.7 \%$ $(S D=6.88 \%)$ of the stimuli in accordance with the category membership predicted by the decision bound. On the implicit task, the participants classified an average of $79 \%$ $(S D=5.2)$ of the stimuli in accordance with the category membership predicted by the decision bound. In both tasks, 11 of the 12 participants had higher classification accuracy when accuracy was defined in terms of the decision bound, rather than in terms of actual category membership.

The participants showed only a small amount of learning during scanning on the novel-implicit task. Overall, the participants achieved $58 \%$ accuracy $(S D=7.6 \%$, range of $41 \%-68 \%)$. On the first scan, accuracy was $55.9 \%$ $(S D=6.7 \%)$, and on the second scan, it was $60.3 \%(S D=$ $8.7 \%)$. Response latency was significantly faster in the verbal task $(M=806 \mathrm{msec})$ than in the implicit task $(M=$ $967 \mathrm{msec}$ ) or in the novel-implicit task $[M=975 \mathrm{msec}$; $F(2,11)=23.6, p<.0001]$.

Debriefing. Ten of the 12 participants were asked after scanning to describe to the experimenter any strategy they had used to distinguished between Categories A and B in the verbal and the implicit tasks. Owing to experimenter oversight, they were not asked to describe strategies used in the novel-implicit task. The reported strategy for the 

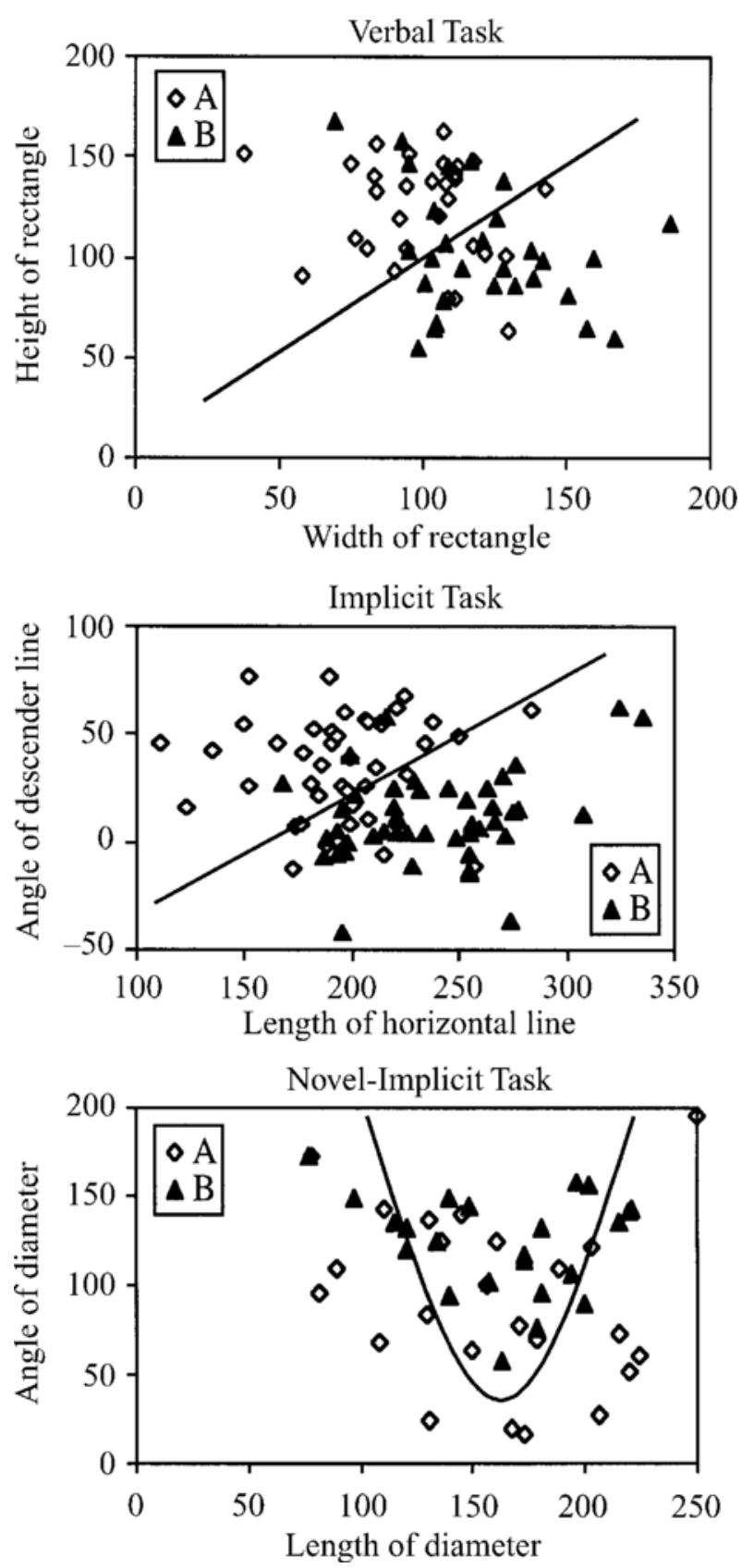

Figure 2. Scatterplots of Category $A$ and B stimuli used in each task, plotted as a function of the features manipulated in each task. The lines superimposed on the scatterplots are the approximate ideal decision bounds for categorization.

verbal task was very consistent across participants. All 10 participants reported a variant of the verbal rule "choose A if the height is greater than the width, B if the width is greater than the height." The participants phrased this rule in different words, including "higher equals A, wider equals B," "A is vertical, B is sideways," and "As have a wider base, lower height; Bs have a narrower base, greater height."
The reported strategies in the implicit task varied across participants. All 10 participants were able to verbally report some perceived regularity of the stimuli and referred to at least one of the two dimensions manipulated. Four participants gave rules that referred to only one of the two dimensions, 3 mentioning the line angle ("A if the angle was less than $30^{\circ}$," "A if the angle was less than $45^{\circ}$," and "A if the angle was in the middle [near vertical] and B otherwise") and 1 mentioning line length ("A if descender was longer than the horizontal line; otherwise, B"). Five participants gave pairs of rules, one for each of the manipulated dimensions. They generally stated that they applied both rules and indicated which rule prevailed if there was a conflict. These five rule sets were the following: (1) "If the line projecting out from the long horizontal line was pointing more downward than to either side (greater than $45^{\circ}$ ), then group $\mathrm{B}$. When the projection was longer than half of the length of the horizontal line, then group A. The latter rule overruled the former"; (2) "If the angle was less than $45^{\circ}$, then A; otherwise, B. If the descending line was greater than half of the horizontal line, then A; otherwise, $\mathrm{B}$. If the angle was less than $25^{\circ}$, then that overrode the middle rule-A no matter what"; (3) "If the length of the descender was shorter than half the length of the horizontal line, then B. Secondarily, if the angle was close to $90^{\circ}$, then B; otherwise, A"; (4) "In Bs, the descenders were nearer the center, around $80^{\circ}-100^{\circ}$. In As, they were usually more toward the horizontal. But if the horizontal was long and the angle near vertical, it was a $\mathrm{B}$. If the horizontal was relatively short, it was an A, even with a vertical angle"; (5) "Acute angles were As. Acute angles and short lines were Bs. Angles greater than 90 were Bs, unless the horizontal line was longer than the descender, in which case it was an A." The 10th participant stated that he looked at "how close the ends were to each other." It is unclear whether he made this distinction on the basis of the angle, the line length, or some combination.

Participants who reported a single rule referring to one feature for the implicit task performed similarly to participants who reported two or more rules on the implicit task, in terms of both number of blocks to criterion on the implicit task $(M=6.5$ and 6.2, respectively; $t<1.0)$, and percentage correct during scanning on the implicit task $(M=$ $71.8 \%$ and $72.0 \%$, respectively; $t<1.0$ ). Interestingly, the participants who reported using a one-dimensional rule for the implicit task tended to require more blocks to criterion on the verbal task $(M=5.5)$ than did participants who reported a two-dimensional rule $[M=3.6 ; t(7)=$ $1.67, p=.14]$.

\section{Imaging Results}

Striatal activations. All three concept tasks (verbal, implicit, and novel-implicit) showed similar patterns of activation of the basal ganglia, in comparison with baseline, as is shown in Table 2 and Figure 3. Activation was found bilaterally in both the putamen and the caudate nuclei. Within the caudate, activity extended from the anterior portions (the head) to posterior portions (the tail). A regions- 
of-interest (ROI) analysis was performed to compare the percentages of signal change within the caudate and the putamen across conditions, as is shown in Figure 4. ROIs were defined functionally for the right and left caudate and putamen by identifying voxels that were commonly active in all three experimental conditions, in comparison with baseline, using a clusterwide threshold of $p=.0025$ and an extent threshold of 20 voxels. This analysis identified two ROIs, one in the right hemisphere, consisting of 124 voxels, and one in the left hemisphere, consisting of 117 voxels. Comparison of these voxel coordinates with the atlas of Tailarach and Tournoux (1988) verified that these ROIs encompassed the caudate and putamen nuclei. By using the ROIs, the percentage of signal change from the overall mean in each area was calculated separately for each of the 12 subjects in each of the four conditions. In both the left and the right caudate and putamen, percentage of signal change was lower in the baseline task than in the three experimental tasks. A one-way analysis of variance showed a significant effect of task in the right-hemisphere ROI $[F(3,11)=3.41, p<.05]$. The effect did not reach significance in the left-hemisphere ROI $[F(3,11)=1.93$, $p=.14]$.

Occipital activations. Left occipital areas were active in the implicit $>$ baseline and novel-implicit $>$ baseline comparisons, but not in the verbal $>$ baseline comparison. Left occipital lobe activation was also found in both the novel-implicit $>$ verbal and implicit $>$ verbal comparisons (see Table 3). These activations were in left lateral extrastriate visual areas, including parts of Brodmann's Areas (BA) 18, 19, and 37 in the inferior and middle occipital gyri and the inferior temporal gyrus. There was a significant cluster of activation in homologous areas of the right middle occipital gyri in the novel-implicit $>$ verbal comparison, with subthreshold activation in these areas in the implicit $>$ baseline, novel-implicit $>$ baseline, and implicit $>$ verbal comparisons.

Medial frontal activations. The three concept-learning tasks showed similar activations, in comparison with baseline, in bilateral medial frontal areas, including the anterior cingulate. In addition, in both the verbal $>$ novel-implicit and the verbal $>$ implicit comparisons, there was more ac-

Table 2

Coordinates of Clusters of Activation Showing Significantly Higher Activity in the Concept-Learning Tasks Than in the Baseline Task

\begin{tabular}{|c|c|c|c|c|c|}
\hline Region & $k$ & $x$ & $y$ & $z$ & $\mathrm{BA}$ \\
\hline \multicolumn{6}{|c|}{ Verbal > Baseline } \\
\hline \multirow[t]{3}{*}{ Right striatum } & 664 & 24 & -2 & -2 & \\
\hline & & 18 & -8 & 0 & \\
\hline & & 28 & 12 & -2 & \\
\hline \multirow[t]{3}{*}{ Left striatum } & 441 & -22 & -8 & 0 & \\
\hline & & -14 & -10 & -4 & \\
\hline & & -28 & 4 & 0 & \\
\hline \multirow[t]{3}{*}{ Right anterior cingulate } & 999 & 20 & 40 & 0 & 24 \\
\hline & & 16 & 20 & 32 & 32 \\
\hline & & 32 & 30 & 20 & 32 \\
\hline \multirow[t]{3}{*}{ Left anterior cingulate } & 335 & -26 & 34 & 20 & 32 \\
\hline & & -20 & 28 & 30 & 32 \\
\hline & & -28 & 24 & 28 & 32 \\
\hline \multicolumn{6}{|c|}{ Implicit > Baseline } \\
\hline \multirow[t]{3}{*}{ Right striatum and anterior cingulate } & 529 & 28 & 32 & -2 & 24 \\
\hline & & 24 & 22 & -2 & \\
\hline & & 24 & 38 & 2 & 32 \\
\hline \multirow[t]{3}{*}{ Left striatum } & 250 & -14 & 2 & 0 & \\
\hline & & -24 & -12 & 2 & \\
\hline & & -16 & -8 & 4 & \\
\hline \multirow[t]{2}{*}{ Bilateral superior medial frontal } & 242 & 4 & 30 & 38 & 8 \\
\hline & & -10 & 32 & 40 & 8 \\
\hline \multirow[t]{3}{*}{ Left occipital } & 609 & -28 & -86 & 0 & 18 \\
\hline & & -34 & -88 & -10 & 18 \\
\hline & & -48 & -72 & -2 & 19 \\
\hline \multicolumn{6}{|c|}{ Novel-Implicit $>$ Baseline } \\
\hline \multirow[t]{3}{*}{ Bilateral striatum and anterior cingulate } & 3,642 & 22 & 34 & 16 & 32 \\
\hline & & -10 & 8 & 52 & 6 \\
\hline & & 26 & 34 & -2 & 24 \\
\hline \multirow[t]{3}{*}{ Left occipital } & 388 & -42 & -82 & 6 & 19 \\
\hline & & -34 & -88 & -8 & 18 \\
\hline & & -30 & -82 & 2 & 18 \\
\hline
\end{tabular}

Note-All clusters reached an uncorrected significance level of $p=.01$ and an extent threshold of 20 voxels. For each cluster, coordinates are given for the maximally activated voxel and up to two local maxima. $k$, number of voxels in cluster; BA, Brodmann's areas; $x, y, z$, MNI coordinates. 


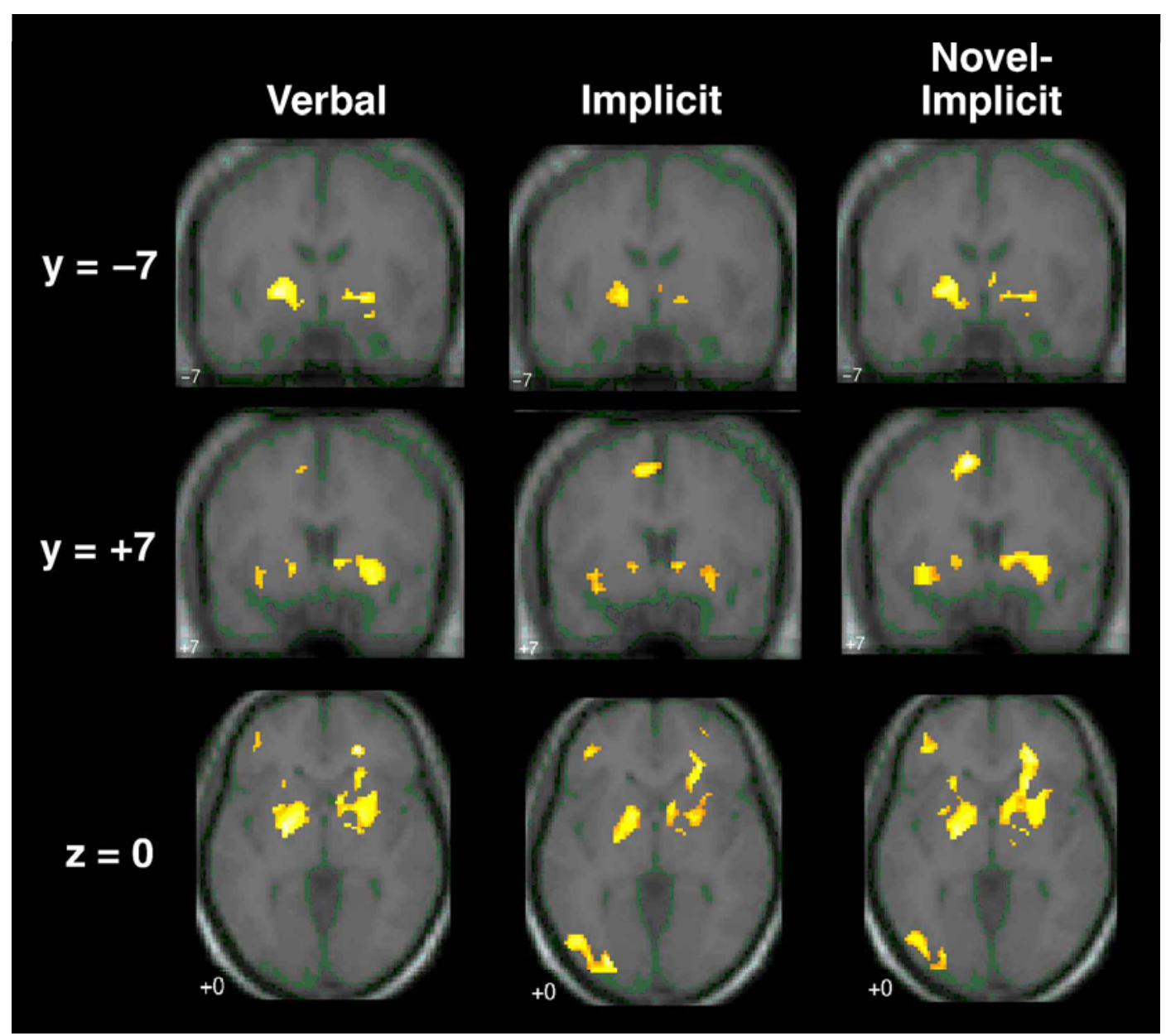

Figure 3. Average striatal and occipital activation in verbal, implicit, and novel-implicit concept-learning tasks in comparison with baseline. Activations are overlaid on the average anatomical image formed from 8 of the participants; the anatomical images of the remaining 4 participants were lost owing to computer disk failure following functional image normalization. The top two rows are overlays onto coronal sections at $y=+7$ and $y=-7$; the bottom row are overlays onto axial sections at $z=0$.

tivity in the verbal condition in the medial inferior frontal lobe. This area encompassed parts of BAs 10 and 11.

\section{DISCUSSION}

The present study found very similar patterns of striatal activation during three concept-learning tasks that differed in participant expertise and verbalizability. Striatal activation did not differ between well-learned classification tasks (verbal and implicit) and a novel classification task (novel-implicit). Striatal activation also did not differ between easily verbalized (verbal) and implicit (implicit and novel-implicit) tasks. However, lateral occipital areas were more active in the implicit tasks (implicit and novelimplicit) than in the verbal task.

Ashby et al.'s (1998) theory of concept learning predicts that the verbal task should activate the head of the caudate, which interacts with prefrontal regions, and that the implicit task should activate the tail of the caudate, which interacts with inferior temporal regions. This pattern of acti- vation was not found in the present study: Both the verbal and the implicit tasks activated the striatum broadly, including both the head and the tail of the caudate. However, their model predicts that the two systems will be in competition during classification, and it is possible that both systems are active during each concept-learning task. Ashby et al.'s theory further predicts that visual form processing areas corresponding to monkey area TE should be active in implicit tasks because of the interaction between area TE and the tail of the caudate in the visual processing loop of the basal ganglia. This study did find activation in lateral occipital and inferior temporal areas in the implicit tasks (implicit and novel-implicit), but not in the verbal task. These areas do fall within the ventral visual pathway that processes form; further research is needed to determine whether they can be considered a human analogue of monkey TE.

Across studies, the striatum has been reported as active in some, but not all, visual concept-learning tasks. Other tasks in which striatal activity has been reported include a 

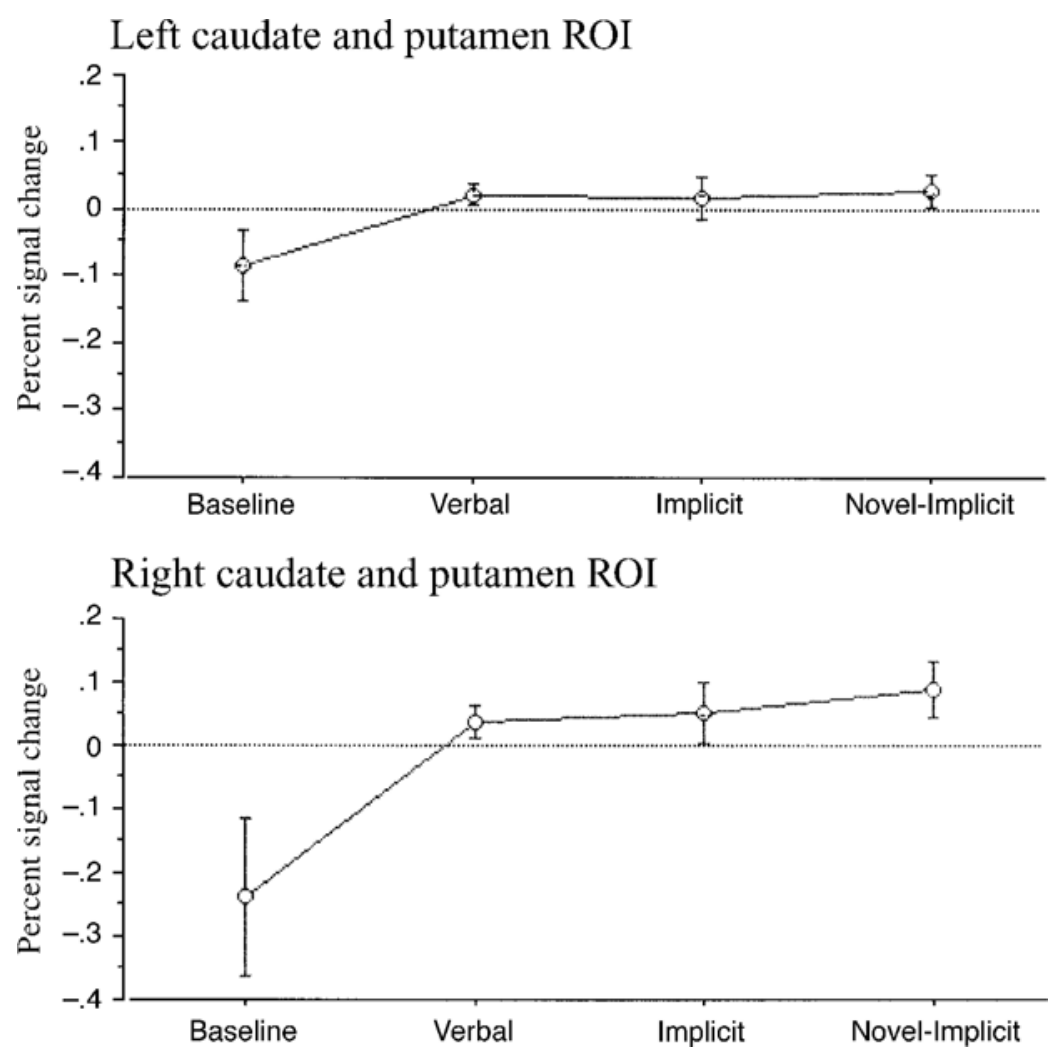

Figure 4. Percentages of signal change in the left caudate and putamen (above) and the right caudate and putamen (below) across the baseline and concept-learning tasks. See the text for details of this region of interest (ROI) analysis.

probabilistic classification weather prediction task (Poldrack et al., 1999), a task in which subjects learned, via hypothesis generation and feedback, the correct rule to use to select one of two items (Rao et al., 1997), and the WCST (Monchi et al., 2001). No striatal activity was reported in a study in which rule-based classifications was compared with memory-based classification (Smith et al., 1998) and in a study in which learning to classify exemplars formed as low-distortion exemplars of two concept prototypes was examined (Seger, Poldrack, et al., 2000).

What factors may be responsible for these differences in striatal activation? One characteristic in common between several of the studies in which striatal activity was found is that there is a probabilistic relationship between the stimulus and its category membership or outcome, rather than an invariant relationship. In the Poldrack et al. (1999) study, each stimulus pattern was associated with both outcomes across trials, but with different probabilities. In the present study, the overlap between categories was such that $20 \%$ of the time, a stimulus was more similar to the prototype of the other category than to that of its own category. However, in the Smith et al. (1998) study, the rule used by participants was always correct in predicting concept membership, and in the Seger, Poldrack, et al. (2000) study, the two concept prototypes were quite dissimilar, and participants had little uncertainty about category membership.
A second common characteristic is that participants often receive negative feedback in the studies that find striatal activity. In the Poldrack et al. and the present studies, error rates were high because of the probabilistic nature of the tasks, as was described above. In the Rao et al. (1997) study, the rules were invariant, but participants had to learn them via feedback and often were wrong in the early trials. Monchi et al. (2001), in an event related f MRI study of the WCST, found that caudate activity was specifically associated with receiving negative feedback in the first trials after the rule was changed. Further research is required to determine whether striatal activity is attributable either to the probabilistic nature of the concept structure or to receiving and processing negative feedback.

In the present study, there was more lateral occipital lobe activity in the novel-implicit and implicit tasks than in the verbal and baseline tasks. This pattern of activation implies that the tasks that involved implicit learning had greater visual processing demands than did the verbal task. In the verbal task, the participants needed only to check whether the relative lengths of two dimensions matched their verbal rule, whereas in the implicit tasks, the participants had to perform a more involved scrutiny of the stimulus features. Animal research has found that ventral visual stream areas (area TE in monkeys) provide necessary input to striatal learning systems and that per- 
Table 3

Coordinates of Clusters of Activation Showing Significantly Different Activity in Comparisons Between Concept-Learning Tasks

\begin{tabular}{|c|c|c|c|c|c|}
\hline Region & $k$ & $x$ & $y$ & $z$ & BA \\
\hline \multicolumn{6}{|c|}{ Implicit > Verbal } \\
\hline Left occipital & 308 & $\begin{array}{l}-40 \\
-46 \\
-32\end{array}$ & $\begin{array}{l}-90 \\
-78 \\
-88\end{array}$ & $\begin{array}{r}2 \\
-2 \\
-12\end{array}$ & $\begin{array}{c}18 \\
19 \\
18,19\end{array}$ \\
\hline \multicolumn{6}{|c|}{ Verbal > Implicit } \\
\hline Medial prefrontal & 392 & $\begin{array}{r}-8 \\
6 \\
-6\end{array}$ & $\begin{array}{l}56 \\
44 \\
38\end{array}$ & $\begin{array}{r}-2 \\
-10 \\
-8\end{array}$ & $\begin{array}{c}10 \\
10,11 \\
32\end{array}$ \\
\hline \multicolumn{6}{|c|}{ Novel-Implicit > Verbal } \\
\hline Left occipital & 1,180 & $\begin{array}{l}-38 \\
-40 \\
-26\end{array}$ & $\begin{array}{l}-86 \\
-76 \\
-86\end{array}$ & $\begin{array}{r}8 \\
-2 \\
-8\end{array}$ & $\begin{array}{l}19 \\
18 \\
18\end{array}$ \\
\hline Right occipital & 249 & $\begin{array}{l}36 \\
44 \\
48\end{array}$ & $\begin{array}{l}-88 \\
-72 \\
-64\end{array}$ & $\begin{array}{r}0 \\
-6 \\
-4\end{array}$ & $\begin{array}{l}18 \\
19 \\
19\end{array}$ \\
\hline \multicolumn{6}{|c|}{ Verbal > Novel-Implicit } \\
\hline \multicolumn{6}{|c|}{ No significant clusters of activation } \\
\hline \multicolumn{2}{|c|}{ No significant clusters of activation } & & & & \\
\hline \multicolumn{6}{|c|}{ Implicit > Novel-Implicit } \\
\hline No significant clus & & & & & \\
\hline
\end{tabular}

formance on concurrent discrimination tasks is impaired when these areas are lesioned (Buffalo et al., 1999; Buffalo et al., 1998).

The pattern of occipital activation (greater for implicit tasks than for the verbal task) found in this study is compatible with a study by Seger, Prabhakaran, et al. (2000), who found lateral occipital activity during implicit judgments of artificial grammar strings, but not during explicit recognition judgments. In addition, Smith et al. (1998) found higher occipital lobe activation in memory-based classification (which may rely on mechanisms similar to those for the implicit task) than in rule-based classification (comparable to the verbal condition). Several studies have shown occipital lobe activity differences for concept members versus nonmembers; these studies do indicate a role of the occipital lobe in classification but cannot be directly compared with the present study, because the present study did not compare category members and nonmembers. The pattern across studies is that when implicit classification judgments are made, occipital activity is lower for category members than for nonmembers (Aizenstein et al., 2000; Reber, Stark, \& Squire, 1998a, 1998b), whereas when recognition judgments are made, occipital activity is higher for category members than for nonmembers (Aizenstein et al., 2000; Reber et al., 1998a).

In the present study, the structures underlying the concepts were chosen so that the concept-learning tasks would differ in terms of how much the participants could verbalize about their decision-making processes. In the verbal task, two compatible features were manipulated, and their mean values were chosen so that a simple verbal rule could be used to describe the ideal decision bound between categories. In the implicit tasks (implicit and novel-implicit), incompatible features were manipulated, making it impossible to form a single simple verbal rule that would allow classification with a high degree of accuracy. The debriefing results indicate that the participants did indeed differ in the accuracy and completeness of the verbal strategies they reported for the implicit and the verbal tasks. For the verbal task, all the participants reported using the same rule as that intended by the investigators. For the implicit task, the participants were able to verbalize some of the regularities present in the stimuli, but these regularities differed between participants and did not accurately and completely describe the decision bound between the categories.

How do the differences between the verbal and the implicit tasks used in this study correspond with those in other tasks that find differences between explicit and implicit processing? First, it should be noted that the explicit/ implicit distinction is typically used in memory research to differentiate between tasks that rely on the hippocampaldiencephalic memory system (explicit) and tasks that can be learned independently of this memory system (implicit); however, the present study was not designed to examine differences in dependence on the hippocampal-diencephalic memory system, and the tests used here have not been used with persons with global amnesia who have damage 
to the hippocampal-diencephalic memory system. Alternatively, the terms implicit and explicit have been used to refer to differences in participants' conscious awareness during learning. Participants are aware that they are learning in explicit tasks and have more verbalizable knowledge of what they have learned and what factors are influencing their performance. Implicit tasks are associated with relatively less verbalizable knowledge; however, many studies have shown that participants do acquire some correlated verbalizable knowledge during implicit tasks, and cases of implicit learning with no accompanying verbalizable knowledge are rare (Seger, 1994b; for varying perspectives on this issue, see Berry, 1997). The present study did show differences in the participants' ability to verbalize their classification strategies. During debriefing, the participants consistently gave a verbal equivalent of the ideal decision bound for the verbal task. In the implicit task, the participants were able to verbalize at least limited rules describing their performance; however, the rules verbalized were not sufficient to support the high level of classification accuracy that these participants displayed. Thus, there were differences in verbalizability between the verbal and the implicit tasks, as was intended. However, it should be noted that in the implicit task, as in many other implicit learning tasks, the participants did have some verbalizable knowledge that correlated with the features of the categories manipulated. Thus, the implicit task can be considered to be relatively more implicit than the verbal task but does not meet strict criteria for implicit learning that require that the learning be completely inaccessible to conscious awareness (Berry, 1997).

Previous concept-learning studies (Poldrack et al., 1999; Rao et al., 1997; Seger, Poldrack, et al., 2000; Smith et al., 1998) have invariably found activity in lateral frontal and inferior parietal areas thought to subserve working memory networks (Smith \& Jonides, 1997). However, in the present study, no significant activity in these areas was found in any of the three concept-learning tasks, in comparison with baseline. Even when a low threshold was used ( $p=.1$, uncorrected, with an extent threshold of $k=0$ ), there was still no activity apparent in these areas or in any areas other than those reported in Tables 2 and 3. It is unclear why frontal and parietal areas were not active in the present experiment. The baseline task was a simple task involving viewing a stimulus and making a motor response, similar to that used in several studies that did find significant frontal activity (Poldrack et al., 1999; Seger, Poldrack, et al., 2000). One possibility is that pretraining in this study led participant performance to be relatively more automatic than in other concept-learning studies; increased automaticity of performance has been shown to reduce or eliminate frontal activity (Raichle et al., 1994).

Inferior medial frontal areas were more active in the verbal task than in the novel-implicit and implicit tasks. This portion of the medial frontal lobe is often active in neuroimaging studies, typically in baseline or relatively simpler tasks, in comparison with experimental or relatively more complex tasks (Gusnard \& Raichle, 2001; Shulman et al., 1997). The verbal task was easier than the novelimplicit and implicit tasks, as is indicated by the significantly faster response times in the verbal task. Thus, this activation may reflect extraneous thought processes during the less demanding task. It should be noted that subthreshold activity in this area was seen in the baselinenovel-implicit and the baseline-implicit comparisons as well.

In conclusion, we found activity in the striatum, both caudate and putamen, in three concept-learning tasks. Activity in the striatum did not differ across tasks, indicating that the striatum is recruited in classification regardless of expertise (implicit and verbal vs. novel-implicit), verbalizability (verbal vs. implicit), and general difficulty of the classification task (verbal vs. implicit and novel-implicit). These results provide convergent evidence that the striatum plays a role in visual discrimination and stimulusoutcome learning in humans, as well as in other animals.

\section{REFERENCES}

Aggleton, J. P., Shaw, C., \& Gaffan, E. A. (1992). The performance of postencephalitic amnesic subjects on two behavioural tests of memory: Concurrent discrimination learning and delayed matching-tosample. Cortex, 28, 359-372.

Aizenstein, H. J., MacDonald, A. W., Stenger, V. A., Nebes, R. D., Larson, J. K., Ursu, S., \& Carter, C. S. (2000). Complementary category learning systems identified using event-related functional MRI. Journal of Cognitive Neuroscience, 12, 977-987.

Alexander, G. E., DeLong, M. R., \& Strick, P. L. (1986). Parallel organization of functionally segregated circuits linking basal ganglia and cortex. Annual Review of Neuroscience, 9, 357-381.

Ashby, F. G., Alfonso-Reese, L. A., Turken, A. U., \& Waldron, E. M. (1998). A neuropsychological theory of multiple systems in category learning. Psychological Review, 105, 442-481.

Ashby, F. G., \& GotT, R. E. (1988). Decision rules in the perception and categorization of multidimensional stimuli. Journal of Experimental Psychology: Learning, Memory, \& Cognition, 14, 3353.

Ashby, F. G., \& Maddox, W. T. (1992). Complex decision rules in categorization: Contrasting novice and experienced performance. Journal of Experimental Psychology: Learning, Memory, \& Cognition, 18, 50-71.

Berman, K. F., Ostrem, J. L., Randolph, C., Gold, J., Goldberg, T. E., Copula, R, Carson, R. E., Herscovitch, P., \& Weinberger, D. R. (1995). Physiological activation of a cortical network during performance of the Wisconsin card sorting task: A positron emission tomography study. Neuropsychologia, 33, 1027-1046.

Berns, G. S., Cohen, J. D., \& Mintun, M. A. (1997). Brain regions responsive to novelty in the absence of awareness. Science, 276, 12721275.

BERRY, D. C. (1997). How implicit is implicit learning? New York: Oxford University Press.

Buffalo, E. A., Ramus, S. J., Clark, R. E., Teng, E., Squire, L. R. \& ZolA, S. M. (1999). Dissociation between the effects of damage to perirhinal cortex and area TE. Learning \& Memory, 6, 572-599.

Buffalo, E. A., Stefanacci, L., Squire, L. R., \& Zola, S. M. (1998). A reexamination of the concurrent discrimination learning task: The importance of anterior inferotemporal cortex, area TE. Behavioral Neuroscience, 112, 3-14.

Caltagirone, C., Carlesimo, A., Nocentini, U., \& Vicari, S. (1994). Defective concept formation in Parkinsonians is independent from mental deterioration. Journal of Neurology, Neurosurgery, \& Psychiatry, 52, 334-337.

Channon, S., Jones, M.-C., \& Stephenson, S. (1993). Cognitive strate- 
gies and hypothesis testing during discrimination learning in Parkinson's disease. Neuropsychologia, 31, 75-82.

Cohen, J. D., MacWhinney, B., Flatt, M., \& Provost, J. (1993). PsyScope: An interactive graphic system for designing and controlling experiments in the psychology laboratory using Macintosh computers. Behavioral Research Methods, Instruments, \& Computers, 25 , 257-271.

Elliott, R., \& Dolan, R. J. (1999). Differential neural responses during performance of matching and nonmatching to sample tasks at two delay intervals. Journal of Neuroscience, 19, 5066-5073.

Fernandez-Ruiz, J., Wang, J., Aigner, T. G., \& Mishin, M. (2001). Visual habit formation in monkeys with neurotoxic lesions of the ventrocaudal neostriatum. Proceedings of the National Academy of Sciences, 98, 4196-4201.

Friston, K. J., Ashburner, J., Frith, C. D., Poline, J. B., Heather, J. D., \& FrackowIaK, R. S. J. (1995). Spatial registration and normalization of images. Human Brain Mapping, 3, 165-189.

Friston, K. J., Worsley, K. J., \& Frackowiak, R. S. J. (1995). Statistical parametric maps in functional imaging: A general linear approach [Abstract]. Human Brain Mapping, 2, 189.

Friston, K. J., Worsley, K. J., Frackowiak, R. S. J., Mazziotta, J. C., \& Evans, A. C. (1994). Assessing the significance of focal activations using their spatial extent. Human Brain Mapping, 1, 210-220.

GAFFAN, D., \& EACOTT, M. J. (1995). Visual learning for an auditory secondary reinforcer by macaques is intact after uncinate fascicle section: Indirect evidence for the involvement of the corpus striatum. European Journal of Neuroscience, 7, 1866-1871.

Glover, G. H., \& Pauly, J. M. (1992). Projection reconstruction techniques for reduction of motion effects in MRI. Magnetic Resonance Medicine, 28, 275-289.

Goldberg, T. E., Berman, K. F., Fleming, K., Ostrem, J., Van Horn, J. D., Esposito, G., Mattay, V. S., Gold, J. M., \& Weinberger, D. R. (1998). Uncoupling cognitive workload and prefrontal cortical physiology: A PET rCBF study. NeuroImage, 7, 296-303.

Grafton, S. T., Hazeltine, E., \& Iv RY, R. (1995). Functional mapping of sequence learning in normal humans. Journal of Cognitive Neuroscience, 7, 497-510.

Gusnard, D. A., \& Raichle, M. E. (2001). Searching for a baseline: Functional imaging and the resting human brain. Nature Reviews Neuroscience, 2, 685-694.

Hazeltine, E. Grafton, S. T., \& Ivry, R. (1997). Attention and stimulus characteristics determine the locus of motor-sequence encoding. Brain, 120, 123-140.

Holmes, A. P., \& Friston, K. J. (1998). Generalisability, random effects and population inference. NeuroImage: Abstracts of the 4th International Conference on Functional Mapping of the Human Brain, 7, S754, 136.

Honda, M., Deiber, M.-P., Ibanez, V., Pascual-Leone, A., Zhuang, P., \& Hallett, M. (1998). Dynamic cortical involvementin implicit and explicit motor sequence learning: A PET study. Brain, 121, 21592173.

JaCKson, G. M., JaCkson, S. R., Harrison, J., Henderson, L, \& KenNARD, C. (1995). Serial reaction time learning and Parkinson's disease: Evidence for a procedural learning deficit. Neuropsychologia, 33, 577-593.

Jacobs, D. H., Shuren, J., \& Heilman, K. M. (1995). Impaired perception of facial identity and facial affect in Huntington's disease. Neurology, 45, 1217-1218.

KNOPMAN, D., \& NisSEN, M. J. (1991). Procedural learning is impaired in Huntington's disease: Evidence from the serial reaction time task. Neuropsychologia, 29, 245-254.

Knowlton, B. K., Mangels, J. A., \& Squire, L. R. (1996). A neostriatal habit learning system in humans. Science, 273, 1399-1402.

Knowlton, B. K., Squire, L. R., \& Gluck, M. A. (1994). Probabilistic classification learning in amnesia. Learning \& Memory, 1, 106-120.

Knowlton, B. K., Squire, L. R., Paulsen, J. S., Swerdlow, N. R., Swenson, M., \& Butters, N. (1996). Dissociations within nondeclarative memory in Huntington's disease. Neuropsychology, 10, 538548.

Lawrence, A. D., Sahakian, B. J., \& Robbins, T. W. (1998). Cognitive functions and corticostriatal circuits: Insights from Huntington's disease. Trends in Cognitive Sciences, 2, 379-388.
Middleton, F. A., \& Strick, P. L. (1996). The temporal lobe is a target of output from the basal ganglia. Proceedings of the National Academy of Sciences, 93, 8683-8687.

MinK, J. W. (1996). The basal ganglia: Focused selection and inhibition of competing motor programs. Progress in Neurobiology, 50, 381425.

Mishin, M., Malamut, B., \& Bachevalier, J. (1984). Memories and habits: Two neural systems. In G. Lynch, J. L. McGaugh, \& N. M. Weinberger (Eds.), Neurobiology of learning and memory (pp. 65-77). New York: Guilford.

Monchi, O., Petrides, M., Petre, V., Worsley, K., \& Dagher, A. (2001). Wisconsin card sorting revisited: Distinct neural circuits participating in different stages of the task identified by event-related functional magnetic resonance imaging. Journal of Neuroscience, 21, 7733-7741.

Nagahama, Y., Fukuyama, H., Yamauchi, H., Matsuzaki, S., KoNISHI, J., ShIBASAKI, H., \& KimURA, J. (1996). Cerebral activation during performance of a card sorting test. Brain, 119, 1667-1675.

OsCar-Berman, M., \& Zola-Morgan, S. M. (1980). Comparative neuropsychology and Korsakoff's syndrome: II. Two-choice visual discrimination learning. Neuropsychologia, 18, 513-525.

Packard, M. G., Hirsch, R., \& White, N .M. (1989). Differential effects of fornix and caudate nucleus lesions on two radial maze tasks: Evidence for multiple memory systems. Journal of Neuroscience, $\mathbf{9}$, 1465-1472.

PACKARD, M. G., \& McGaugh, J. L. (1992). Double dissociation of fornix and caudate nucleus lesions on acquisition of two water maze tasks: Further evidence for multiple memory systems. Behavioral Neuroscience, 106, 439-446.

Poldrack, R. A., Prabhakaran, V., Seger, C. A., \& Gabrieli, J. D. E. (1999). Striatal activation during cognitive skill learning. Neuropsychology, 13, 564-574.

Ragland, J. D., Gur, R. C., Glahn, D. C., Censits, D. M., Smith, R. J., Lazarev, M. G., Alavi, A., \& Gur, R. E. (1998). Frontotemporal cerebral blood flow change during executive and declarative memory tasks in schizophrenia: A positron emission tomography study. $\mathrm{Neu}$ ropsychology, 12, 399-413.

Raichle, M. E., Fiez, J. A., Videen, T. O., MacLeod, A. M. K., Pardo, J. V., Fox, P. T., \& Petersen, S. E. (1994). Practice-related changes in human functional anatomy during non-motor learning. Cerebral Cortex, 4, 8-26.

Rao, S. M., Bobholz, J. A., Hammeke, T. A., Rosen, A. C., Woodley, S. J., Cunningham, J. M., Cox, R. W., Stein, E. A., \& Binder, J. R. (1997). Functional MRI evidence for subcortical participation in conceptual reasoning skills. NeuroReport, 8, 1987-1993.

Reber, P. J., Stark, C. E. L., \& SQuire, L. R. (1998a). Contrasting cortical activity associated with category memory and recognition memory. Learning \& Memory, 5, 420-428.

Reber, P. J., Stark, C. E. L., \& Squire, L. R. (1998b). Cortical areas supporting category learning identified using functional MRI. Proceedings of the National Academy of Sciences, 95, 747-750.

SEger, C. A. (1994a). Criteria for implicit learning: De-emphasize conscious access, emphasize amnesia. Behavioral \& Brain Sciences, 17, 421-422.

SegER, C. A. (1994b). Implicit learning. Psychological Bulletin, 115, 163 196.

Seger, C. A., Poldrack, R. A., Prabhakaran. V., Zhao, M., Glover, G., \& Gabrieli, J. D. E. (2000). Hemispheric asymmetries and individual differences in visual concept learning as measured by functional MRI. Neuropsychologia, 38, 1316-1324.

Seger, C. A., Prabhakaran, V., Poldrack, R. A., \& Gabrieli, J. D. E. (2000). Neural activity differs between explicit and implicit learning of artificial grammar strings: An f MRI Study. Psychobiology, 28, 283-292.

Shulman, G. L., Fiez, J. A., Corbetta, M., Buckner, R. L., Miezin, F. M., Raichle, M. E., \& Petersen, S. E. (1997). Common blood flow changes across visual tasks: II. Decreases in cerebral cortex. Journal of Cognitive Neuroscience, 9, 648-663.

Smith, E. E., \& Jonides, J. (1997). Working memory: A view from neuroimaging. Cognitive Psychology, 33, 5-42.

Smith, E. E., Patalano, A. L., \& Jonides, J. (1998). Alternative strategies of categorization. Cognition, 65, 167-196. 
Squire, L. R., Zola-Morgan, S., \& Chen, K. S. (1988). Human amnesia and animal models of amnesia: Performance of amnesic patients on tests designed for the monkey. Behavioral Neuroscience, 102, 210221.

TAlairach, J., \& Tournoux, P. (1988). Co-planar stereotactic atlas of the human brain. New York: Thieme.

TAY LOR, A. E., SAINT-Cyr, J. A., \& LANG, A. E. (1986). Frontal lobe dysfunction in Parkinson's disease. Brain, 109, 845-883.

Teng, E., Stefanacci, L., Squire, L. R., \& Zola, S. M. (2000). Contrasting effects on discrimination learning after hippocampal lesions and conjoint hippocampal-caudate lesions in monkeys. Journal of Neuroscience, 20, 3853-3863.

Wang, J., Aigner, T., \& Mishrin, M. (1990). Effects of neostriatal lesions on visual habit formation in rhesus monkeys. Society for Neuroscience Abstracts, 16, 617.
Weinberger, D. R., Berman, K. F., Iadarola, M., Driesen, N., \& ZEC, R. F. (1988). Prefrontal cortical blood flow and cognitive function in Huntington's disease. Journal of Neurology, Neurosurgery, \& Psychiatry, 51, 94-104.

Willingham, D. B., \& Koroshetz, W. J. (1993). Evidence for dissociable motor skills in Huntington's disease patients. Psychobiology, 21, 173-182.

Wise, S. P., Murray, E. A., \& Gerfew, C. R. (1996). The frontal cortex-basal ganglia system in primates. Critical Reviews in Neurobiology, 10, 317-356.

(Manuscript received April 21,2001;

revision accepted for publication March 10, 2002.) 